$\mathrm{Zu}$ den neueren Behandlungsmethoden zählt das Skinboosting, bei dem stabilisierte, wenig quervernetzte Hyaluronsäure in feinen Tröpfchen großflächig mittels kurzer linearer Technik, Fächer- oder Criss-Cross-Technik injiziert wird. Ziel ist nicht die lokale Faltenkorrektur oder der Volumenaufbau, sondern eine Verbesserung der Hautstruktur.

Die Effekte auf die Hautqualität belegen wissenschaftliche Untersuchungen [1]: Es kommt zu einer Dehnung der extrazellulären Matrix und einer indirekten Stimulation der Fibroblasten, die in der Folge vermehrt Kollagen bilden. Die messbar gesteigerte Bildung von Typ-1-Prokollagen im Behandlungsareal war auch nach einem Jahr noch nachweisbar. Damit verbessern sich Elastizität und Struktur der Haut nachhaltig. Die Patienten sollten aber darauf hingewiesen werden, dass wiederholte Behandlungen sinnvoll und für nachhaltige Effekte erforderlich sind.
Das Skinboosting eignet sich für eine Hautauffrischung im Gesicht, an Hals und Dekolleté sowie an den Händen. Gut geeignet sind Skinbooster Ogilvie zufolge auch für problematische Areale wie Oberlippenfältchen, wenn keine Veränderung in Volumen und Form der Lippen gewünscht wird. Herabgezogene Mundwinkel werden ebenfalls gebessert, eine zusätzliche Volumengabe zur Behandlung einer Nasolabialfalte oder eine Behandlung des Musculus depressor anguli oris mit Botulinumtoxin A kann aber sinnvoll sein. Die Behandlung sollte schrittweise erfolgen, um möglichst natürliche Ergebnisse zu erzielen, empfahl Ogilvie.

Angelika Bauer-Delto

Praktischer Kurs: Neue Behandlungsmethoden der Ästhetischen

Dermatologie

1. Quan T et al. J Invest Dermatol 2013;133:658-67

\title{
Phototherapie soll wichtige Säule dermatologischer Therapien bleiben
}

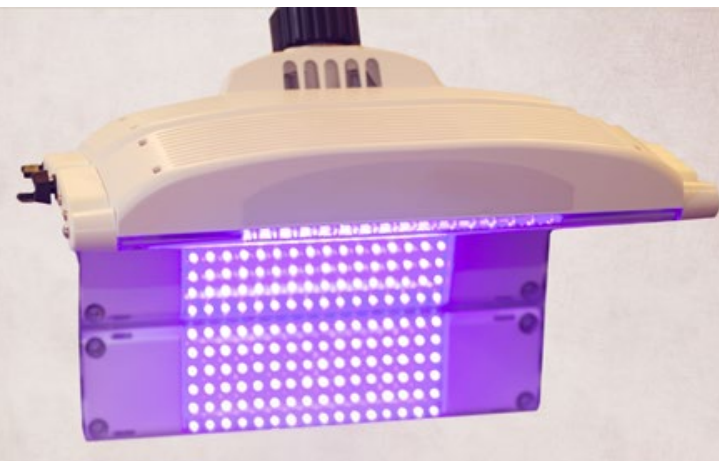

Das Know-how der Phototherpie muss erhalten bleiben.

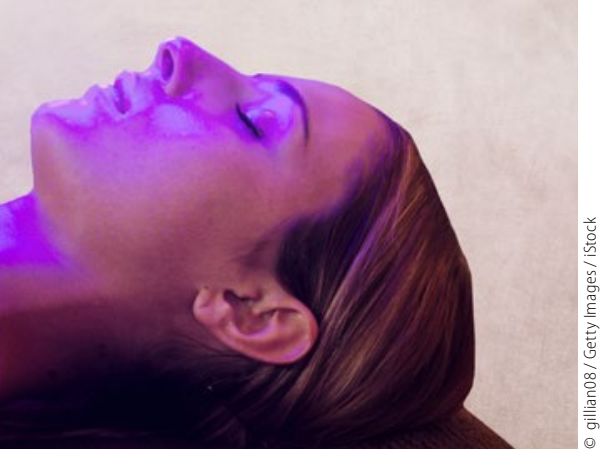

Seit der antiken Heliotherapie hat die Phototherapie einige Höhen und Tiefen erlebt. Doch nicht zuletzt wegen ihrer guten Kombinierbarkeit ist sie neben Salben- und Systemtherapie auch heute noch eine der drei Säulen des dermatologischen Instrumentariums.

Als Königsdisziplin unter den konventionellen Therapien bezeichnete Prof. Percy Lehmann, Wuppertal, die PUVA-Therapie. Ihre Wirkung bei der Psoriasis sei noch immer unschlagbar. Allerdings habe sie den Nachteil, dass zwar die Haut hervorragend behandelt würde, nicht aber die Arthritis. Im Bereich des UV-B-Spektrums hat die Schmalspur-Therapie bei $311 \mathrm{~nm}$ das Breitspektrum abgelöst. Damit kann die Psoriasis viel aggressiver behandelt werden, sodass bei dreimal wöchentlicher Therapie nach zwölf Wochen bei 80 \% der Patienten ein PASI 75 erreicht wird. Diese Wirksamkeit sei vergleichbar mit Infli- ximab und Adalimumab und besser als Etanercept, so Lehmann. Es entstehe viel weniger Erythem als beim Breitspektrum, die Sicherheit sei höher als bei Biologika und die Kosten seien zehnfach geringer. Allerdings ist der Zeitaufwand weitaus größer als bei Biologika und es ist eine hohe Compliance erforderlich. Auch die chronische Form des atopischen Ekzems reagiert gut auf UV-B $311 \mathrm{~nm}$. Die UV-A1-Phototherapie ist evidenzbasiert bei akut exazerbierter atopischer Dermatitis und zirkumskripter Sklerodermie.

Trotz all dieser Erfolge ist die Phototherapie rückläufig. Der Grund hierfür seien „new sexy drugs“, so Lehmann. Jetzt wiederhole sich die nachlassende Beliebtheit der Phototherapie, die in der Kortison- und Antibiotika-Ära beobachtet wurde mit den Biologika. Dabei würden unter PUVA $80 \%$ Clearing bei $90 \%$ der Patienten in vier bis sechs Wochen erreicht, während unter Biologika 60-75\% der Patienten in zwölf Wochen einen PASI 75 erzielten. Und während unter PUVA bei nur $2 \%$ der Patienten das Ergebnis unbefriedigend war, lag diese Quote unter Biologika bei $10-15 \%$. Hinsichtlich der Praktikabilität seien Biologika bei vernünftigem Einsatz allerdings unschlagbar, meinte Lehmann. Es sei ein Traum für jeden Psoriatiker, nur alle vier Wochen eine Spritze zu erhalten, statt dreimal pro Woche zum Arzt zu gehen. Für Langzeitprognosen hinsichtlich der Sicherheit der Biologika sei es allerdings zu früh, warnte der Dermatologe. Außerdem existiere ein ökonomisches Paradoxon: „Moderne Systemtherapien, insbesondere Biologika, verursachen sehr hohe Kosten für die Solidargemeinschaft. Für den Kassenarzt aber ist die Phototherapie aufgrund hoher Anschaffungs- und Wartungskosten sowie inadäquatem Entgelt innerhalb des Budgets unwirtschaftlich. Deshalb wird sie in den Praxen häufig abgebaut." Damit gehe wichtiges Know-how verloren. So appellierte Lehmann an seine Kollegen: „Wir sollten die Phototherapie hegen und pflegen. Im Rahmen der Mono-, Kombinations- und Rotationstherapien wird sie ihren zentralen Platz, unter Berücksichtigung der hochinteressanten pharmakologischen Neuentwicklungen, in den nächsten Jahrzehnten behalten.“

Dr. Christine Starostzik

"Hat die Phototherapie noch eine Zukunft?": Vortrag von Prof. Percy Lehmann 